\title{
FUNDAMENTOS TEÓRICOS DO PROCESSO DE FORMAÇÃO DE CONCEITOS E SUAS IMPLICAÇÕES PARA O ENSINO E APRENDIZAGEM DE QUÍMICA
}

\begin{abstract}
RESUMO: A problemática do processo de formação de conceitos encontra explicação nas diversas correntes filosóficas, dentre as quais podem ser citadas a empirista e a materialista dialética. A primeira acredita que o esquema formativo dos conceitos deva se apoiar nas experiências sensoriais e na capacidade de representação dos objetos, enquanto a segunda atribui grande importância aos conhecimentos historicamente construídos, destacando os aspectos relacionados tanto a sua antogênese quanto a sua filogênese. Nesse sentido, o presente trabalho pretende contribuir com as pesquisas em Educação Química, na medida em que busca uma maior aproximação entre as investigações de cunho filosófico-psicológico e as de caráter didáticopedagógico, particularmente quanto ao estudo das vias de desenvolvimento dos conceitos científicos em situações de ensino e aprendizagem.
\end{abstract}

Palavras-chave: conceitos, ensino e aprendizagem, Química.

\section{THEORETICAL BACKGROUND OF THE PROCESS OF CONCEPT FORMATION AND ITS IMPLICATION FOR TEACHING AND LEARNING IN CHEMISTRY}

ABSTRACT: The problematic of the concepts formation process finds explanation in the several philosophical currents, among which the empiricist and the dialectical-materialistic can be mentioned. The former believes that the concepts formative scheme must be supported by the sensorial experience and by the object representation capacity, while the second gives great importance to the historically built knowledge, highlighting the aspects related to its antogenesis as well as to its phylogenesis. In this direction, the present work aims to contribute to the research in chemical education, as it searches an approximation between the psychological-philosophical and the didactic-pedagogic investigation, particularly in relation to the study of the scientific concepts development on teaching and learning. Keywords: concepts, teaching and learning, Chemistry.

\author{
Rodrigo Garrett da Costa* \\ Liliana Maria Passerino** \\ Milton Antonio Zaro ${ }^{* * *}$
}

\footnotetext{
*Doutorando em Informática na Educação pela Universidade Federal do Rio Grande do Sul (PGIE/UFRGS) Professor do curso de Licenciatura em Química do Instituto Federal de Educação Ciência e Tecnologia Fluminense, campus Campos - Centro e-mail: rgarrett@iff.edu.br **Doutora em Informática na Educação pela Universidade Federal do Rio Grande do Sul (PGIE/UFRGS). Professora pesquisadora da UFRGS atuando na Pós-Graduação em Educação (PPGEDU)

e na Pós-Graduação em Informática na Educação (PGIE). email: liliana@pgie.ufrgs.br ***Doutor em Engenharia Metalúrgica pela Universidade Federal do Rio Grande do Sul (UFRGS). Pósdoutor pela Universidade Federal de Santa Catarina. Professor/pesquisa dor convidado da Universidade Federal do Rio Grande do Sul, no Programa de PósGraduação em Informática na Educação (PGIE/UFRGS) e-mail: zaro@ufrgs.br
} 


\section{INTRODUÇÃO}

A teoria empírica de pensamento, desenvolvida pelo filósofo John Locke, viveu o seu auge nos séculos XVIII e XIX, tendo exercido grande influência sobre a psicologia e a didática durante esse período. Ainda no início do século XXI, percebe-se a grande influência das ideias empiristas, sobretudo na escolarização formal, em áreas do conhecimento tradicionalmente marcadas pela experimentação - como a Química, a Física e a Biologia.

Uma das contribuições dessa corrente filosófica, particularmente importante para o ensino e aprendizagem de Química, diz respeito ao processo de formação e desenvolvimento de conceitos. Sob o ponto de vista das ideias empiristas, pode-se afirmar que estes últimos sejam

\footnotetext{
[...] fruto de um processo original de estratificação das imagens sensoriais umas sobre as outras, durante o qual desaparecem os traços semelhantes dos objetos, enquanto que as características comuns se reforçam mutuamente, formando as representações e conceitos gerais, bem como as suas palavras correspondentes. (LEONTIEV, 1964, p. 86 citado por DAVYDOV, 1972, p. 78)
}

Essa concepção acerca do processo de formação de conceitos constitui um dos pilares que sustentam as práticas tradicionais de ensino de Química. Em função da natureza empírica e muitas vezes abstrata dessa área do conhecimento, costuma-se observar, com frequência, a adoção de práticas educacionais pautadas na ampliação da capacidade de representação dos estudantes.

Sendo assim, acredita-se que a observação dos traços sensoriais dos objetos seja capaz de provocar, por si só, a ampliação da faculdade de representação dos estudantes, fato que justificaria a grande importância atribuída às suas experiências individuais cotidianas. Estas últimas seriam determinantes para o estabelecimento e a ampliação de vínculos associativos entre as classes de objetos, assegurando a existência de um elemento essencial para o processo de conceitualização - a generalização ${ }^{1}$. Sob esse ponto de vista, "cada objeto é visto como um conjunto de indícios estáveis e independentes entre si. Ao se confrontarem esses indícios, observa(m)-se aquele(s) que aparece(m) em todos os objetos dados e que são os traços comuns" (DAVYDOV, 1972, p.75).

Não obstante, esse modo de interpretar o processo de formação de conceitos, defendido tanto pela lógica formal quanto pela didática tradicional, é alvo de diversas críticas. A principal delas corresponde à afirmação de que as ideias empiristas se restringem à descrição das vias de formação dos conceitos espontâneos, não sendo capazes de nos fornecer uma explicação satisfatória quanto ao processo de formação dos conceitos mais complexos, conhecidos como teóricos ou científicos.

Alguns pesquisadores que se apoiam na filosofia do materialismo dialético (como Davydov, Galperin, Leontiev e Vigotski), ao discordarem das ideias empi- 
Fundamentos teóricos do processo de formação de conceitos

e suas implicações para o ensino e aprendizagem de Química

ristas quanto ao processo de formação dos conceitos teóricos, apontam para os seguintes problemas existentes nessa linha de pensamento:

- pressupõe-se a necessidade da existência de traços sensoriais concretos, para que sejam destacados os indícios gerais e se estabeleçam as classificações que originam os conceitos;

- necessita-se das identificações dos objetos sensoriais concretos, com posterior inclusão nas classes, para que ocorra o surgimento dos conceitos.

Para Davydov (1972), a obrigatoriedade de manipulação das qualidades sensoriais dos objetos e fenômenos funciona como uma espécie de limitação à compreensão dos conhecimentos teóricos, uma vez que estes operam mediante a análise dos seus vínculos internos. Esse tipo de conhecimento, como é mais estruturado, requer ainda a produção de meios especiais de abstração, análise e generalização, os quais permitem fixar os nexos internos das coisas, ou seja, suas essências. Dessa maneira, acredita-se que o modo de elaboração dos conceitos teóricos seja distinto daquele observado entre os conceitos empíricos, visto que ambos operariam por vias específicas.

$\mathrm{Na}$ concepção filosófica do materialismo dialético, o processo de formação de conceitos (em especial os conceitos teóricos) é tratado como algo bastante complexo. Não se compartilha a ideia de que a ampliação das experiências sensoriais seja o fator determinante para o processo de conceitualização. Ao invés disso, destaca-se a necessidade da existência de certas condições que devem ser favoráveis ao processo de formação de juízos e deduções, abrindo-se a possibilidade de reflexão acerca das propriedades gerais e essenciais dos objetos e fenômenos.

Nota-se que as explicações voltadas ao processo de formação dos conceitos diferem bastante, dependendo do ponto de vista de uma ou outra corrente filosófica. De fato, existe uma quantidade considerável de estudos sobre esse tema, tanto na esfera da Filosofia, quanto da Psicologia e da Didática, o que pode ser atribuído a sua grande importância histórica e científica ao longo dos séculos.

Mesmo assim, não se pode afirmar que o assunto em questão esteja em vias de esgotamento, pois, mesmo no início do século XXI, existem diversas investigações sendo conduzidas com o objetivo de depreender as vias de desenvolvimento dos conceitos, sobretudo nas diversas situações de ensino e aprendizagem.

Nesse sentido, o presente trabalho pretende contribuir com as pesquisas em Educação Química, na medida em que busca uma maior aproximação entre as investigações de cunho filosófico-psicológico e as de caráter didático-pedagógico, particularmente quanto ao estudo das vias de desenvolvimento dos conceitos científicos em situações de ensino e aprendizagem. Para tanto, realizamos uma breve abordagem sobre os principais aspectos filosóficos envolvidos no estudo dos conceitos, e em seguida discutimos algumas questões relacionadas à prática docente e às situações de ensino que operam mediante conceitos teóricos. 


\section{FORMAÇÃO DE CONCEITOS SEGUNDO A LÓGICA FORMAL E A DIDÁTICA TRADICIONAL}

O esquema formativo dos conceitos (com início na percepção, passando pela representação e culminando na conceitualização) pressupõe a existência de alguns elementos constitutivos, os quais obedecem à seguinte ordem: em primeiro lugar, a existência de traços essenciais que permitam distinguir uma classe de objetos das demais; em segundo lugar, a verbalização do seu significado; por último, o conhecimento desse significado, que possui caráter abstrato e ocorre independentemente da existência de uma imagem concreta. Na opinião de Davydov (1972), esse processo no qual se alcança o conceito por meio da representação, partindose da percepção, equivaleria ao trânsito do sensorial, concreto e singular ao mental, abstrato e geral.

Os conhecimentos concretos são aqueles que representam os objetos em si e se caracterizam por serem observáveis e perceptíveis através dos nossos sentidos. Por outro lado, os conhecimentos abstratos apresentam propriedades que não podem ser perceptíveis nos objetos, visto que são fruto de abstrações; além disso, são designados por meio da palavra e integram a rede de conceitos.

$\mathrm{Na}$ didática tradicional, compreende-se que o processo de abstração ocorra em função da acumulação de percepções e representações, ou seja, o pensamento abstrato teria maior possibilidade de ocorrer em pessoas que fossem mais providas de experiências sensoriais e de representações. Sob esse ponto de vista, deve-se procurar adotar métodos de ensino que ofereçam aos estudantes a oportunidade de comparação de um grande número de objetos ou fenômenos afins, pelos quais se possam analisar quais são suas propriedades comuns e também as distintas. Isso significa que as propostas de ensino que se baseiam no princípio da lógica formal devem dispor de formas e materiais diversificados para estudo, que englobem grandes quantidades de variantes combinatórias das propriedades afins, destacando suas características comuns.

$\mathrm{Na}$ perspectiva empirista, o ponto de partida para a formação de conceitos é a percepção sensorial dos objetos singulares, os quais posteriormente são utilizados para explicar os fenômenos observados. Sendo assim, é atribuída grande importância às experiências anteriores, quase sempre apoiadas em observações visuais, táteis e auditivas, as quais serviriam como base para o processo de obtenção de novos conhecimentos.

\section{FORMAÇÃO DE CONCEITOS SEGUNDO O MATERIALISMO DIALÉTICO E A TEO- RIA HISTÓRICO-CULTURAL}

As primeiras investigações sobre o processo de formação dos conceitos, elaborados com base nessa filosofia marxista, foram desenvolvidas por Vigotski (2001). Apesar da importância dos seus trabalhos para o campo da psicologia, po- 
Fundamentos teóricos do processo de formação de conceitos

e suas implicações para o ensino e aprendizagem de Química

de-se afirmar que a teoria histórico-cultural não contemplou, de início, as ideias relativas ao meio educacional, na medida em que teve como foco a formação dos conceitos nas crianças.

Apesar disso, suas ideias funcionaram como uma espécie de marco teórico, fazendo com que outros pesquisadores se interessassem pelo tema, dando prosseguimento a suas descobertas, porém sob uma perspectiva mais voltada ao processo de ensino e aprendizagem. Como exemplo, podem-se citar os trabalhos de Leontiev (1978) e Davydov (1972, 1988), no campo da Teoria da Atividade; de Galperin $(1959,1986)$, com a Teoria da Assimilação por Etapas das Ações Mentais; e posteriormente a noção de Perfil Conceitual proposta por Mortimer (1995, 1996, 2000).

Diferentemente da filosofia empírica, que reconhece o pensamento do indivíduo na sua generalidade, desconsiderando seus aspectos individuais, a filosofia dialética do pensamento constitui-se como um "processo objetivo da atividade humana, movimento da civilização e da sociedade como autênticos sujeitos do pensamento" (KOPNIN, 1969 citado por DAVYDOV, 1972, p. 153). Sob esse ponto de vista, atribui-se grande importância aos conhecimentos historicamente construídos, o que resulta na necessidade de se conhecerem não apenas os aspectos relacionados à antogênese do conhecimento científico, mas também aqueles relativos a sua filogênese. Além disso, acredita-se na intervenção da natureza sobre o objeto de conhecimento humano, na medida em que ela é incorporada à atividade objetiva transformadora e produtiva. Nesse processo, o homem (ser social) apropria-se dos objetos por meio de atividades práticas, as quais são sensório objetivas, e não através da contemplação passiva (DAVYDOV, 1972, p.294).

Quanto às formas de compreensão dos objetos da atividade cognoscitiva, Davydov atesta a existência de dois níveis de pensamento: o pensamento empírico e o pensamento teórico. O primeiro é fruto direto das observações humanas e está relacionado a suas atividades prático-materiais e sócio-políticas. Ele corresponde às representações sensoriais, como os sistemas simbólicos e os sinais, os quais podem criar condições para atividades mais complexas, culminando no processo de generalização.

O pensamento teórico, por sua vez, opera mediante conceitos científicos e caracteriza-se pela presença de experimentos mentais. Além disso, pressupõe o domínio dos fenômenos objetivamente inter-relacionados, dentro de um sistema integrado de conceitos. Essa dinâmica que se estabelece entre um conceito e outro num dado contexto de análise, bem como o trânsito desde um fenômeno ou conceito particular até certo todo, com anulação da sua especificidade - ou seja, sua conexão interna -, é revelada pelo pensamento teórico e científico.

$\mathrm{Na}$ ausência desse sistema, qualquer fenômeno passa a ser considerado como mero objeto, fruto da observação empírica. Contrariamente às constatações verificadas por meio do pensamento empírico, que são efetivas, observáveis e desarticuladas, no pensamento teórico existem predominantemente dependências 
intrínsecas e substanciais, impossíveis de serem observadas diretamente pelos sentidos.

No pensamento teórico, o concreto aparece duas vezes: como ponto inicial da contemplação e representação do conceito, mas também como resultado mental das associações de abstrações. Essa característica dialética do concreto permite ao homem elaborar, na forma de conceito, os dados de contemplação e representação, os quais por meio de conexões internas possibilitam-lhe revelar sua essência.

Para Davydov (1972), o pensamento teórico se caracteriza pela ascensão do abstrato ao concreto, movimento que só é possível graças aos processos de abstração e generalização. Segundo o autor, não se trata de pensar apenas abstratamente com um conjunto de proposições fixas, mas de se desenvolver relações gerais que possam ser percebidas em muitas situações específicas.

Enquanto na abstração tem-se a retenção mental das relações reais que determinam a integridade dos fenômenos, na generalização são estabelecidos os nexos reais dessas relações particulares, agora desarticuladas, com os outros fenômenos da base da mesma.

A abstração corresponderia à análise do objeto, livre de traços e peculiaridades não essenciais, enquanto a generalização teórica consistiria na redução dos diversos fenômenos semelhantes a suas respectivas bases teóricas (DAVYDOV, 1972). A presença desses dois fenômenos constitui a base do processo de formação dos conceitos científicos, ou seja,

[...] La generalización científica [...] es la generalización que llega al conocimiento de la esencia, de la regularidad de desarrollo de las cosas [...] Lo general es la ley, la esencia de los fenómenos singulares, o sea, algo cualitativamente distinto con respecto a la simples suma de indicios de las cosas sueltas. (ROZENTAL, 1960, p. 216-217 citado por DAVYDOV, 1972, p. 354)

Se, por um lado, a filosofia empírica possui como princípio a valorização das qualidades sensoriais dos objetos e fenômenos como base para se alcançar o conhecimento, por outro, o materialismo dialético entende que o pensamento teórico é subjacente ao sensorial ou tangível, possuindo a propriedade de transformar o mundo circundante. Para Davídov (1988, p. 124-125),

[...] é necessário sublinhar que o fundamento e a fonte de todos os conhecimentos do homem sobre a realidade são as sensações e as percepções, dados sensoriais. Porém, os resultados da atividade dos órgãos dos sentidos do homem são expressos por este em forma verbal, que acumula a experiência de outras pessoas.

Dessa forma, o trânsito tanto da matéria à consciência como da sensação ao pensamento pode ser expresso numa atividade mental que extrapola as qualidades sensoriais observáveis, uma vez que 
Fundamentos teóricos do processo de formação de conceitos

e suas implicações para o ensino e aprendizagem de Química

[...] La actividad mental, a nivel superior, permite descubrir nuevos aspectos del objeto, "encubiertos" desde la óptica de las posibilidades del nivel inferior. La forma de expresión del conocimiento teórico aparece a este respecto como "modelo" del objeto en el sentido único del mencionado [...] término de que las operaciones con el mismo permiten dilucidar determinados aspectos del objeto que nos es posible esclarecer mediante el manejo [...] directo de este último. (SHVYRIEV, 1966, p. 131 citado por DAVYDOV, 1972, p. 365)

O processo de formação e desenvolvimento dos conceitos científicos, para Vigotski (2001), pressupõe a existência de três elementos psicológicos fundamentais: (1) a formação de relações entre os conceitos, ou melhor, do sistema de conceitos; (2) a tomada de consciência da própria atividade mental; e (3) o surgimento de relações especiais que o indivíduo adquire com os objetos, o que the permite compreender melhor a essência destes últimos.

Quanto ao primeiro elemento destacado, considera-se que ele seja o responsável por permitir que os conceitos surjam e se organizem. Já a tomada de consciência das operações mentais corresponde a sua reconstituição (mental), visando-se à operação discursiva - processo que está diretamente relacionado com a generalização dos processos psíquicos. Uma vez que essa tomada de consciência reflita na própria atividade do sujeito, diz-se que está ocorrendo um processo especial de generalização, presente nos conceitos científicos e nas formas superiores de pensamento humano.

\section{ALGUMAS IMPLICAÇÕES PARA O ENSINO DE CONCEITOS QUÍMICOS}

A natureza microscópica e muitas vezes abstrata, característica dos conhecimentos químicos, costuma provocar, entre os estudantes, dificuldades na aprendizagem das diversas leis e conceitos. Além disso, existe o fato de a linguagem química ser essencialmente simbólica, o que pressupõe a necessidade de uma grande capacidade de abstração e generalização.

O estudo da Química, principalmente nas universidades, caracteriza-se pela abordagem de uma grande quantidade de conhecimentos teóricos. Por meio deles, são destacadas as qualidades internas dos objetos e fenômenos, ou seja, seus atributos não perceptíveis diretamente, visto que são fruto de deduções e relações teóricas. Sendo assim, para que sua aprendizagem seja efetiva, propomos a estruturação das situações de ensino, tendo-se como base o princípio do método dedutivo de pensamento, ou seja, partindo-se do conhecimento geral para o particular e do abstrato para o concreto.

Nessa perspectiva, devem ser abordadas primeiramente as leis e princípios, para somente em seguida serem analisadas as diversas situações particulares. Uma vez compreendidas as leis gerais, conseguem-se interpretar com mais propriedade os conceitos e fenômenos subjacentes, o que possibilitaria aos alunos identificá-los futuramente nas diversas situações específicas.

Por todos esses fatores, acreditamos que tanto o ato de ensinar, como o 
de aprender conceitos químicos não seja tarefa fácil. Se por um lado os educadores precisam aperfeiçoar constantemente a sua prática de sala de aula, de modo a acompanhar a velocidade das transformações científicas, tecnológicas e sociais que se estabelecem, por outro, os estudantes precisam se sentir estimulados e dispostos a aprender. Isso equivale à busca de mecanismos que visem ao estabelecimento das chamadas “operações especiais”, as quais, segundo Davydov (1972), são fundamentais para a promoção do pensamento teórico e caracterizam-se por revelar o conteúdo das abstrações, generalizações e conceitos constitutivos de uma determinada área ou de qualquer das suas partes essenciais.

Exemplos de operações especiais são a idealização ${ }^{2}$ e a capacidade de se operar com símbolos, sejam estes materiais ou gráficos. Os símbolos funcionam como um meio de reprodução da realidade, representada não por meio da própria natureza, mas das propriedades dos objetos que a compõem. Davydov (1972) esclarece que os vários sistemas de símbolos são formas de padronização e, portanto, de idealização dos objetos materiais. Sendo assim, a transferência dos objetos ao plano mental, bem como a utilização mediatizada dos símbolos para revelar a essência das coisas, equivale ao movimento rumo à reprodução teórica da realidade.

A modelização é mais uma forma de idealização, nesse caso visual e simbólico-sinalizadora, que apresenta grande aplicação na Química e na Física (assim como em outras áreas das ciências) e cuja estruturação se baseia principalmente na função mediatizadora dos signos. Seu objeto essencial - o modelo ${ }^{3}$ - pode apresentar-se sob duas formas distintas: os materiais e os mentais (SHTOFF, 1966 citado por DAVYDOV, 1972). Enquanto os primeiros voltam-se às atividades práticas, os últimos se relacionam às transformações mentais.

A importância das atividades de modelização para a formação do pensamento teórico, na opinião de Davydov, está relacionada ao fato de que

\begin{abstract}
Los modelos son una forma de abstracción científica de índole especial, en la que as relaciones esenciales del objeto destacadas al efecto están consolidadas en nexos y relaciones gráfico-perceptibles y representables de elementos materiales o signalizadores. Entraña una original unidad de lo singular y lo general, en la que se adelantan a primer plano los momentos de carácter general y esencial. [...] son fruto de una compleja actividad cognoscitiva, que incluye sobre todo la elaboración mental del material sensorio inicial, su depuración de elementos casuales, etc. [...] actúan como fruto y como medio de la ejecución de esa actividad. (DAVYDOV, 1972, p. 315)
\end{abstract}

Os modelos materiais subdividem-se em três tipos: existem os que refletem as particularidades espaciais dos objetos, aqueles dotados de semelhanças físicas com o objeto original, e também os modelos matemáticos, os quais refletem as propriedades estruturais dos objetos. Já os modelos mentais, por sua vez, subdividem-se em dois tipos: os ícone-figurativos e os modelos de signos. Estes últimos envolvem as fórmulas ou equações matemáticas e as representações quí- 
Fundamentos teóricos do processo de formação de conceitos

e suas implicações para o ensino e aprendizagem de Química

micas, através das quais se torna possível a observação da estrutura e do comportamento das diversas transformações químicas.

Justi \& Driel (2005) esclarecem que o uso correto de atividades de modelização no ensino de Química deve ser apoiado em formas de abordagem que suscitem entre alunos a oportunidade de criar, expressar e testar seus próprios modelos. Já Greca e Santos (2005) destacam a necessidade de se estimular a explicação e a exploração dos fenômenos, processos e ideias abstratas, bem como o desenvolvimento da capacidade de representação dos estudantes.

\section{CONSIDERAÇÕES FINAIS}

Este manuscrito procurou fazer uma breve apresentação das ideias defendidas pelo empirismo e pelo materialismo dialético, acerca da formação de conceitos. Ao analisar as diferentes posições adotadas por essas duas correntes filosóficas a respeito desse tema, chamamos a atenção para alguns pontos conflitantes, os quais produzem implicações diretas para o ensino, em particular para o ensino de Química.

Dentre as características observadas nessa área do conhecimento, mencionamos a necessidade de se operar com conceitos teóricos, os quais possuem como propriedades essenciais: (1) a operação mediante a análise dos seus vínculos internos; (2) a impossibilidade de elaboração sem os meios especiais de abstração, análise e generalização; (3) a capacidade de reflexão acerca das propriedades gerais e essenciais dos objetos e fenômenos; (4) o domínio dos fenômenos objetivamente inter-relacionados, dentro de um sistema integrado de conceitos; e (5) a tomada de consciência da própria atividade mental, com posterior exteriorização através de sistemas de símbolos e sinais.

Com base nessas premissas, foram feitas algumas recomendações a respeito do processo de ensino e aprendizagem de conceitos químicos, particularmente quanto à prática docente e às situações de ensino que operam mediante conceitos teóricos. Nesse contexto, destacamos a importância das atividades de modelização, uma vez que elas permitem aos estudantes explorarem a função mediatizadora dos signos, além de desenvolverem a capacidade de representação.

\section{NOTAS}

${ }^{1}$ Segundo Davydov (1972), esse processo se caracteriza pela busca do comum e pela nomeação de certos invariantes em determinado conjunto de objetos. O resultado desse processo consiste na capacidade de se separarem os traços comuns e portanto gerais, para que, em seguida, sejam identificados os objetos como pertencentes ou não a determinada classe.

${ }^{2}$ Segundo Davídov (1988), essa operação constitui um dos aspectos da atividade objetivo-prática, a reprodução, nela, das formas universais das coisas. Tal reprodu- 


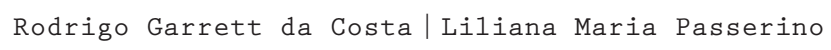

ção tem lugar na atividade laboral das pessoas como peculiar experimento objetivo-sensorial. Logo, esse experimento adquire, cada vez mais, um caráter cognoscitivo, permitindo às pessoas passarem, com o tempo, a realizar os experimentos mentalmente.

${ }^{3}$ Definido por Shtoff (1966) citado por Davydov (1972) como "...un sistema concebido mentalmente o realizado en forma material, que, reflejando o reproduciendo el objeto de la investigación, es capaz de substituirlo de modo que su estudio nos dé nueva información sobre dicho objeto."

\section{BIBLIOGRAFIA}

DAVÍDOV, V. V. La enseñanza escolar y el desarrollo psíquico. Moscou: Progreso, 1988.

DAVYDOV, V. V. Tipos de generalización en la enseñanza. La Habana: Editorial Pueblo y Educación, 1972.

GALPERIN, P. I. "Desarrollo de las investigaciones sobre la formación de acciones mentales". In: Ciencia Psicológica en la URSS, Moscou, v. 1, 1959.

GALPERIN, P. "Sobre el método de formación por etapas de las acciones intelectuales". In: Antología de la Psicología Pedagógica y de las edades. La Habana: Editorial Pueblo y Educación, 1986, pp. 114-118.

GRECA, I. M.; SANTOS, F. M. T. Dificuldades da generalização das estratégias de modelagem em ciências: o caso da física e da química. In: Investigações em Ensino de Ciências, V10(1), pp. 31-46, 2005

JUSTI, R.; DRIEL, J. “A Case Study of the Development of a Beginning Chemistry Teacher's Knowledge about Models and Modelling”. Research in Science Education, p. 197 219, 2005.

KOPNIN, P. V. Filosofskie idiéi V. I. Lenina i lóguika / Las ideas filosóficas de V. I. Lenin y la lógica. Moscú, Náuka, 1969, citado por DAVYDOV, V. V. Tipos de generalización en la enseñanza. La Habana: Editorial Pueblo y Educación, 1972.

LEONTIEV, A. N. Myshlenie / El pensamiento. In: Voprosy filosofii. 1964, núm. 4, citado por DAVYDOV, V. V. Tipos de generalización en la enseñanza. La Habana: Editorial Pueblo y Educación, 1972.

LEONTIEV, A. N. O desenvolvimento do psiquismo. Lisboa: Livros Horizonte, 1978.

MORTIMER, E. F. “Conceptual change or conceptual profile change?”. Science \& Education. London, v. 4, n. 3, 1995.

MORTIMER, E. F. "Construtivismo, mudança conceitual e ensino de ciências: para onde vamos?”. Revista Investigações em Ensino de Ciências, V1(1), pp. 20-39, 1996.

MORTIMER, E. F. Linguagem e Formação de Conceitos no Ensino de Ciências.v.1. Belo Horizonte: Editora UFMG, 2000. v. 01. 383 p.

ROZENTAL, M. M. Printzipy diallékticheskoi lóginki / Principios de lógica dialéctica, Moskú, Sotzekguiz, 1960, citado por DAVYDOV, V. V. Tipos de generalización en la enseñanza. La Ha- 


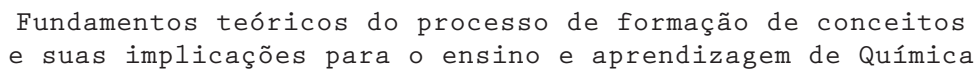

bana: Editorial Pueblo y Educación, 1972.

SHTOFF, V.A. Modelírovanie i filosofia / El modelado y la filosofia. Moscú-Leningrado, Naúka, 1966, citado por DAVYDOV, V. V. Tipos de generalización en la enseñanza. La Habana: Editorial Pueblo y Educación, 1972.

SHVYRIEV, V. S. Neopozitivizm i problemy empiricheskogo obosnovania náuki / El neopositivismo y los problemas de la fundamentación empírica dela ciencia. Moscú, Naúka, 1966, citado por DAVYDOV, V. V. Tipos de generalización en la enseñanza. La Habana: Editorial Pueblo y Educación, 1972.

VIGOTSKI, L. S. A Construção do Pensamento e da Linguagem. Tradução Paulo Bezerra, $1^{\circ}$ ed., São Paulo: Martins Fontes, 2001.

Data de recebimento: 12/04/2011

Data de aprovação: 12/11/2011

Data da versão final: 10/12/2011 